\title{
Isospin non-equilibrium in heavy-ion collisions at intermediate energies
}

\author{
Bao-An $\mathrm{Li}^{a}$ and Sherry J. Yennello ${ }^{b}$ \\ a Cyclotron Institute and Department of Physics \\ b Cyclotron Institute and Department of Chemistry \\ Texas A\&M University, College Station, TX 77843, USA
}

\begin{abstract}
We study the equilibration of isospin degree of freedom in intermediate energy heavy-ion collisions using an isospin-dependent BUU model. It is found that there exists a transition from the isospin equilibration at low energies to nonequilibration at high energies as the beam energy varies across the Fermi energy in central, asymmetric heavy-ion collisions. At beam energies around 55 $\mathrm{MeV} /$ nucleon, the composite system in thermal equilibrium but isospin nonequilibrium breaks up into two primary hot residues with $\mathrm{N} / \mathrm{Z}$ ratios closely related to those of the target and projectile respectively. The decay of these forward-backward moving residues results in the strong isospin asymmetry in space and the dependence of the isotopic composition of fragments on the $\mathrm{N} / \mathrm{Z}$ ratios of the target and projectile. These features are in good agreement with those found recently in experiments at NSCL/MSU and TAMU, implications of these findings are discussed.
\end{abstract}


With the availability of high intensity radioactive beams at several existing and proposed facilities, the isospin degree of freedom in nuclear reactions can be studied in large domains of beam energies and projectile-target combinations. In recent experimental studies of the isotopic composition of intermediate mass fragments from central collisions of ${ }^{40} \mathrm{Cl},{ }^{40} \mathrm{Ar}$ and ${ }^{40} \mathrm{Ca}$ with ${ }^{58} \mathrm{Fe}$ and ${ }^{58} \mathrm{Ni}$ at $E_{\text {beam }} / A=25,35,45$ and $53 \mathrm{MeV}$ at NSCL/MSU and TAMU [1] 3], it was found that at $E_{\text {beam }} / A=25$ and $35 \mathrm{MeV}$ the isotopic ratios ${ }^{9} \mathrm{Be} /{ }^{7} \mathrm{Be},{ }^{11} \mathrm{~B} /{ }^{10} \mathrm{~B}$ and ${ }^{13} C /{ }^{12} C$ increase linearly as a function of the increasing $(N / Z)_{c s}$ ratio of the combined target and projectile system, but are independent of the $\mathrm{N} / \mathrm{Z}$ ratio of the target or projectile. This observation strongly indicates the establishment of isospin equilibration in composite systems formed in these reactions before the emission of fragments. In fact, previous studies on similar systems at a much lower energy showed that the isospin degree of freedom was one of the fastest to equilibrate [4]. The most striking and unexpected feature was observed from the isotopic ratios in central collisions at $E_{\text {beam }} / A=53 \mathrm{MeV}$. It was found that the isotopic ratios depend on the $\mathrm{N} / \mathrm{Z}$ of the target and projectile for entrance target-projectile combinations having the same $(N / Z)_{C S}$ ratio, such as ${ }^{40} \mathrm{Ca}+{ }^{58} \mathrm{Fe}$ and ${ }^{40} \mathrm{Ar}+{ }^{58} \mathrm{Ni}$. Moreover, data at very forward and backward angles also show isotope ratios that are not simply a function of the $(N / Z)_{c s}$. It was found that light fragments at backward angles have a much greater dependence on $(N / Z)_{\text {target }}$, while at forward angles have a much greater dependence on $(N / Z)_{\text {projectile, }}$ and could not be explained simply by the pre-equilibrium emission. These features clearly demonstrate that the isospin degree of freedom is not equilibrated in the reactions at $E_{\text {beam }} / A=53 \mathrm{MeV}$ on the time scale of emission of the fragments.

The above observation has some deep implications on the reaction mechanism leading to multifragmentation. It not only establishes a relative time scale of multifragmentation in these reactions but also indicates that the assumption of isospin equilibrium taken for granted in various statistical models for nuclear multifragmentation at intermediate energies must be improved at least. To our best knowledge, the prominent features observed in these experiments were neither predicted nor observed previously in any model simulations. On the contrary, some models which are able to explain many other aspects of multifragmentation 
and reaction dynamics of intermediate energy heavy-ion collisions are unable to explain these features. In particular, statistical models based on the assumption of isospin and thermal equilibrium of the composite system fail to show any entrance channel effect [1] as one would expect. Calculations using an intranuclear cascade code ISABEL [5] show that the N/Z of the residue is very close to the $\mathrm{N} / \mathrm{Z}$ of the initial combined system [1] and thus also failed to reproduce the features observed at $E_{\text {beam }} / A=53 \mathrm{MeV}$. Although the exact origin of this failure is not so clear to us, one expects that the reaction dynamics at these energies should go beyond the nucleon-nucleon cascade and invoke the nuclear mean field. Therefore, the experimentally observed transition from isospin equilibration to non-equilibration as the beam energy increases from below to above the Fermi energy remains an interesting question for theoretical studies.

In this Letter we report on results of a study on the equilibration of isospin degree of freedom using an isospin-dependent Boltzmann-Uehling-Uhlenbeck (BUU) transport model for heavy-ion collisions at intermediate energies [6,7]. The isospin dependence comes into the model [8] through both the elementary nucleon-nucleon cross sections $\sigma_{12}$ and the nuclear mean field $U$. Here we use the experimental nucleon-nucleon cross sections with the explicit isospin dependence [9]. The isospin dependence resides in the fact that the cross section of neutron-proton collisions is about three times that of neutron-neutron or proton-proton collisions at energies interested here.

The nuclear mean field $U$ including the isospin symmetry term is parameterized as

$$
U\left(\rho, \tau_{z}\right)=a\left(\rho / \rho_{0}\right)+b\left(\rho / \rho_{0}\right)^{\sigma}+\left(1-\tau_{z}\right) V_{c}+C \frac{\rho_{n}-\rho_{p}}{\rho_{0}} \tau_{z}
$$

Here $\rho_{0}$ is the normal nuclear matter density, $\rho, \rho_{n}$ and $\rho_{p}$ are the nucleon, neutron and proton densities, respectively. $\tau_{z}$ equals 1 or -1 for neutrons or protons, respectively, and $V_{c}$ is the Coulomb potential. Corresponding to other, probably more complete forms of Skyrme forces, other forms of parameterization for the mean field are possible [10 12]. Here we use the so called soft nuclear equation of state with the nuclear compressibility $K=200$ $\mathrm{MeV}$. The symmetry term comes from averaging over the constituent two-body forces with 
Heisenberg components proportional to $\left(\vec{\tau}_{i} \cdot \vec{\tau}_{j}\right)$ [13]. Its strength $\mathrm{C}$ can be deduced from experiments (e.g. nuclear symmetry energies, optical potentials for nucleon scatterings, excitations of analogue states in $(\mathrm{p}, \mathrm{n})$ reactions). However, the strength deduced varies significantly from reaction to reaction and also depends on the energy of the nucleon [13]. The parameter $\mathrm{C}$ used in transport model calculations also scatters within a large range [11,14, 15]. In the present study, we use a constant $C=32 \mathrm{MeV}$.

First, to study the energy dependence of the reaction mechanism we show in Fig. 1 the density contours in the reaction plane at $\mathrm{t}=200 \mathrm{fm} / \mathrm{c}$ in head-on collisions of $\mathrm{Ar}+\mathrm{Ni}$ at $E_{\text {beam }} / A=25,35,45$ and $55 \mathrm{MeV}$. The solid contours with $\rho=\rho_{0} / 8$ essentially bound the composite systems or heavy residues formed in the reactions. The dotted contours with $\rho=0.05 \rho_{0}$ are the representatives of free nucleons mainly from pre-thermal-equilibrium emissions. The most interesting feature in Fig. 1 is the formation of two heavy residues in the reaction at $E_{\text {beam }} / A=55 \mathrm{MeV}$. While by following the evolution of the heavy residues until $300 \mathrm{fm} / \mathrm{c}$, we found no breakup happening to the residues formed in the reactions at $E_{\text {beam }} / A=25,35$ and $45 \mathrm{MeV}$.

Thermal equilibrium of the heavy residues can be examined by studying the quadrupole moment $Q_{Z Z}$ defined as

$$
Q_{z z}(t)=\int \frac{d \vec{r} d \vec{p}}{(2 \pi)^{3}}\left(2 p_{z}^{2}-p_{x}^{2}-p_{y}^{2}\right) f(\vec{r}, \vec{p}, t) .
$$

Where $f(\vec{r}, \vec{p}, t)$ is the Wigner function from the BUU model calculations. It is clear that $Q_{z z}=0$ is a necessary but not a sufficient condition for thermal equilibrium. We found that the quadrupole moments of heavy residues formed in the reactions at $E_{\text {beam }} / A=25$, 35 and $45 \mathrm{MeV}$ have been damped to about zero by the time of about $300 \mathrm{fm} / \mathrm{c}$. Since the heavy residue formed in the reaction at $E_{\text {beam }} / A=55$ has already broken up into two pieces at about $200 \mathrm{fm} / \mathrm{c}$, it is interesting to examine more closely the evolution of $Q_{z z}$ in this reaction. This is done in Fig. 2 where $Q_{z z}$ of the heavy residue scaled by its mass number $A_{\text {res }}$ is shown as a function of time. For comparison we also show in this figure the evolution of $Q_{z z}$ for the reaction at $E_{\text {beam }} / A=45$. It is seen that the residues are very close 
to thermal equilibrium at about $200 \mathrm{fm} / \mathrm{c}$ indicated by the very small, decreasing amplitude of the oscillation around zero. In the case of $E_{\text {beam }} / A=55 \mathrm{MeV}$, the oscillation is shifted towards a small positive value indicating a slight longitudinal collectivity or transparency. This finding is actually in very good agreement with the transparency found recently at Ganil for the same reaction system at similar beam energies using the INDRA detector [16].

Are the heavy residues observed above in isospin equilibrium ? To answer this question we show in Fig. 3 the numbers of neutrons and protons in the residues on the left $(Z<0)$ and right $(Z \geq 0)$ side of the origin in the head-on reactions of $\mathrm{Ar}+\mathrm{Ni}$ at $E_{\text {beam }} / A=25,35$, 45 and $55 \mathrm{MeV}$. The solid lines are the numbers of protons from the projectile, while the dotdashed lines are the numbers of neutrons from the projectile which comes in originally from the left. The dashed lines are the numbers of protons from the target, while the dotted lines are the numbers of neutrons from the target which comes in originally from the right in the center of mass frame. It is seen that the numbers of neutrons and protons on both sides undergo damped oscillations. The damping is mainly due to nucleon-nucleon collisions and particle emissions. While the oscillations are due to both the restoring force of the mean field and nucleon-nucleon collisions. At $E_{\text {beam }} / A=25 \mathrm{MeV}$ the neutron and proton numbers on the two sides have become very close to each other and the amplitude of oscillation is becoming rather small by the time of $300 \mathrm{fm} / \mathrm{c}$. It indicates that the heavy residue is very close to the isospin equilibrium characterized by the space-time independent proton and neutron number distributions. At $E_{\text {beam }} / A=35 \mathrm{MeV}$ the oscillation is damped at a higher rate to a distribution of particles close to the isospin equilibrium. The isotopic composition of fragments emitted from the residues of these low energy reactions after about $300 \mathrm{fm} / \mathrm{c}$ would therefore essentially reflect the $(N / Z)_{c s}$ of the initial composite system, and there is little forward-backward asymmetry. These features are in good agreement with those found in the preliminary data of $E_{\text {beam }} / A=25$ and $35 \mathrm{MeV}$ [2, [3].

At higher energies, such as $E_{\text {beam }} / A=45$ and $55 \mathrm{MeV}$, there is little oscillation across the overlapping region between the target and projectile. This is mainly because the incoming momenta of projectile-nucleons and target-nucleons are too high for the mean field and 
nucleon-nucleon collisions to be effective enough during a relatively short reaction time to reverse the directions of motion of many nucleons. As a result, there exists a large isospin asymmetry or non-equilibration at these two energies. In particular, on the left side of the origin the $\mathrm{N} / \mathrm{Z}$ ratio of the residue is more affected by that of the target while on the right side it is more affected by that of the projectile. However, it should be stressed that the N/Z ratios on the two sides are not simply those of the target and projectile but a combination of the two depending on the complicated reaction dynamics. In the case of $E_{\text {beam }} / A=$ $55 \mathrm{MeV}$, at the time of about $200 \mathrm{fm} / \mathrm{c}$ the heavy residue has broken up into two pieces with some longitudinal collectivity. The forward moving residue has an average N/Z ratio of about 7.2/6.0 and an excitation energy of about $8.6 \mathrm{MeV} /$ nucleon. While the backward moving residue has an average $\mathrm{N} / \mathrm{Z}$ ratio of about $21 / 18$ and an excitation energy of about 6.8 $\mathrm{MeV} /$ nucleon.

The proper decay of these primary hot residues is beyond the scope of the BUU model. Nevertheless, one can imagine that the decay of these forward-backward moving hot residues with the different $\mathrm{N} / \mathrm{Z}$ ratios formed in reactions at higher energies will result in a strong isospin asymmetry in space. It is also quite understandable that the isotopic composition of fragments emitted from these residues will have much greater dependence on the $\mathrm{N} / \mathrm{Z}$ ratios of the target and projectile, but not much on the $(N / Z)_{c s}$ of the initial composite systems. Indeed, this expectation is further evidenced in our systematic studies on the system ${ }^{40} \mathrm{~S}+{ }^{58} \mathrm{Zn},{ }^{40} \mathrm{Ca}+{ }^{58} \mathrm{Fe}$ and ${ }^{40} \mathrm{Ti}+{ }^{58} \mathrm{Cr}$ at the same beam energies. These features are in good agreement with the indication of the experimental data at $E_{\text {beam }} / A=53 \mathrm{MeV}$. Furthermore, the properties of the primary residues calculated in the above are useful as inputs for a hybrid model study to compare with the data more quantitatively. Although there is no global isospin equilibration in reactions at higher energies, it may not be such a bad approximation to assume the establishment of isospin equilibrium in the two primary residues formed at $E_{\text {beam }} / A=55 \mathrm{MeV}$. One can thus couple the BUU model with statistical models to compare more quantitatively with the experimental data. These studies are now in progress and results will be published elsewhere. 
In summary, we have studied the question of isospin equilibration in intermediate energy heavy-ion collisions using the isospin-dependent BUU model. We found that there exists a transition from the isospin equilibration to non-equilibration as the beam energy increases from below to above the Fermi energy in central, asymmetric heavy-ion collisions. At beam energies around $55 \mathrm{MeV} /$ nucleon, the composite system in isospin non-equilibrium breaks up into two forward-backward moving hot residues with $\mathrm{N} / \mathrm{Z}$ ratios more closely related to those of the target and projectile on the time scale of thermal equilibrium. These features are in good agreement with that found in recent experiments at NSCL/MSU and TAMU.

We would like to thank W. Bauer, C. M. Ko and J. Randrup for helpful discussions. We are also grateful to W. U. Schröder and L.G. Sobotak for communications on the subject of this study. This work was supported in part by the NSF Grant No. PHY-9212209, DOE Grant FG05-86ER40256 and the Robert A Welch Foundation under Grants A-1110 and A1266. One of us (SJY) also acknowledge support from an NSF National Young Investigator Award. 


\section{REFERENCES}

[1] S.J. Yennello, B. Young, J. Yee, J.A. Winger, J. S. Winfield, G.D. Westfall, A. Vander Molen, B.M. Sherrill, J. Shea, E. Norbeck, D.J. Morrissey, T. Li, E. Gualtieri, D. Craig, W. Benenson and D. Bazin, Phys. Lett. B321 (1994) 15.

[2] S. J. Yennello, J.A. Winger, H. Johnston, T. White, E. Gualtieri, D. Craig, S. Hannuschke, J. Yee, R. Pak, A. Vander Molen, W. Benenson, G.D. Westfall, T. Li, W.J. Liope, D. J. Morrissey, J. S. Winfield and M. Steiner, TAMU Prograss in Research, P. I-7, 1994.

[3] H. Johnston, J. Winger, T. White, B. Hurst, D. O’Kelly and S. J. Yennello, TAMU Progress in Research, P. I-6, 1994.

[4] B. Gatty et al., Z. Phys. A273 (1975) 65.

[5] Y. Yariv and Z. Frankel, Phys. Rev. C26 (1982) 2138.

[6] G.F. Bertsch and S. Das Gupta, Phys. Rep. 160, 189 (1988).

[7] W. Bauer, C.K. Gelbke, and S. Pratt, Ann. Rev. Nucl. Part. Sci. 42 (1992) 77.

[8] B.A. Li and J. Randrup, manuscript in preparation.

[9] Total cross sections for reactions of high energy particles, A. Baldini, V. Flaminio, W.G. Moorhead and D.R.O. Morrison, (Springer-Verlag, Berlin), 1988.

[10] B. Remaud, C. Grëgoire, F. Sëbille and P. Schuck, Nucl. Phys. A488 (1988) 423c.

[11] M. Farine, T. Sami, B. Remaud and F. Sebille, Z. Phys. A339 (1991) 363.

[12] L. G. Sobotka, Phys. Rev. C50 (1994) R1272.

[13] P.E. Hodgson, Ch.9.3, Nuclear reactions and nuclear structure, (Clarendon press, Oxford), 1971.

[14] M. Betty, G.F. Bertsch, W.G. Lynch and M. Tohyama, Phys. Rev. C40 (1989) 1685. 
[15] P. Danielewicz, Phys. Rev. C46 (1992) 2002.

[16] B. Borderie, private communication.

\section{FIGURE CAPTIONS}

Fig. 1 Density contours in the reaction plane at $\mathrm{t}=200 \mathrm{fm} / \mathrm{c}$ for head-on collisions of Ar $+\mathrm{Ni}$ at $E_{\text {beam }} / A=25,35,45$ and $55 \mathrm{MeV}$, respectively. The solid contours are for $\rho=\rho_{0} / 8$, while the dotted lines are for $\rho=0.05 \rho_{0}$.

Fig. 2 The evolution of the quadrupole moment per nucleon of heavy residues formed in the head-on collisions of $\mathrm{Ar}+\mathrm{Ni}$ at $E_{\text {beam }} / A=45$ and $55 \mathrm{MeV}$.

Fig. 3 The numbers of neutrons and protons in the residue with $\rho \geq \rho_{0} / 8$ on the left $(Z<0)$ and right $(Z \geq 0)$ side of the origin in the head-on reactions of $\mathrm{Ar}+\mathrm{Ni}$ at $E_{\text {beam }} / A=25,35,45$ and $55 \mathrm{MeV}$ respectively. The solid lines are the numbers of protons from the projectile, while the dotdashed lines are the numbers of neutrons from the projectile. The dashed lines are the numbers of protons from the target, while the dotted lines are the numbers of neutrons from the target. 
This figure "fig1-1.png" is available in "png" format from: http://arxiv.org/ps/nucl-th/9505015v1 
This figure "fig1-2.png" is available in "png" format from: http://arxiv.org/ps/nucl-th/9505015v1 
This figure "fig1-3.png" is available in "png" format from: http://arxiv.org/ps/nucl-th/9505015v1 